\title{
God Doesn't Always Shave with Occam's Razor - Learning When and How to Prune
}

\author{
Hilan Bensusan \\ School of Cognitive and Computing Sciences \\ University of Sussex \\ Falmer, Brighton \\ BN1 9QH \\ UK \\ phone:+44 1273678524 \\ fax:+44 1273671320 \\ email:hilanb@cogs.susx.ac.uk
}

God doesn't always shave with Occam's razor. Malcom Dixon, quoted in

New Scientist 23/4/94 p.51.

\begin{abstract}
The work shows how a meta-learning technique can be successfully applied to decide when to prune, how much pruning is appropriate and what the best pruning technique is for a given learning task.
\end{abstract}

\section{Introduction: the simplicity issue}

'All truth is simple' - is that not a compound lie? Friedrich Nietzsche

Pruning decision trees sometimes prevents overfitting and compensates for the presence of noise in the training set. Often, however, it oversimplifies the trees missing most of their predictive potential. This work makes the assumption that similar problems are likely to require similar pruning patterns and proposes a meta-learning approach to select among various pruning alternatives.

Simplicity has always been seen as somehow connected with successful inductions. Among the many possible rationales for the Occam's principle - everything else being equal, prefer the simplest hypotheses -, one that is solely based on inductive considerations (and not, for example, on the assumption that the world is simple) is the one that claims that simpler hypotheses enjoy greater prior probability. This idea has inspired the minimal encoding principles that explore the balance between simplicity and consistency as an Occam consequence of the balance between prior and posterior probabilities. These principles have been taken farther than the Occam's razor for they consider that even when everything else is not equal, simplicity still matters. In the context of decision tree induction, the Occam's principle is often used to justify a preference for simpler trees. Apart from general theoretical challenges to this idea, recent experimental work has called into question the general use of a simplicity soft bias $[6,8]$. There were various theoretical attempts to establish conditions under which simpler hypotheses are better (see for example [4]). This work provides an experimental approach to the appropriateness of simplicity biases. 


\section{The meta-learning system}

The Entrencher system learns to choose the best learning bias for a learning task among the ones provided in a bias pool ${ }^{1}$. Therefore, it performs a kind of meta-learning. It contrasts with other methods (see [2]) because it uses induction on training problem descriptions. THE ENTRENCHER uses the decision tree generated from the training set and related information to describe a learning task. ThE ENTRENCHER then performs a supervised meta-learning on a set of classified problems divided into training and test sets. The system acts as follows:

1. Applies all the learners in the bias pool to the training problems and tests their performance;

2. Classifies the problems in terms of the best performing bias;

3. Applies a meta-learning procedure to the classified training set of problems, generating a bias classifier;

The performance of the system is tested by assessing the average accuracy achieved by the bias classifier in a set of test problems ${ }^{2}$ The system then comprises the following components:

A baseline learner generates consistent hypotheses that are the working representations of the problems.

A problem descriptor generates values for the descriptor vector.

A bias pool manager classifies the training problems by accuracy and simplicity ${ }^{3}$.

A meta-learner generates a bias classifier.

The Entrencher uses a decision tree baseline learner - similar to a nonpruning C4.5 [7]. The descriptor vector consists of the following real-valued descriptors: Tree nodes per attribute, tree nodes per instance, the average strength of support of each tree leaf, difference in goodness-as-a-splitting-point between the attributes, maximum depth of the tree, number of repeated nodes, shape of the tree, number of leaves divided by tree shape, balance of the tree, number of subtrees with more than two (possibly internal) nodes repeated in the tree. The shape of the tree by the probability of arriving at each leaf given a randomly chosen path from the root to the leaf. This probability $p\left(N_{i}\right)$ of arriving at node $N_{i}$ among the $m$ sibling nodes from the ancestor $N_{A}$ is given by $p\left(N_{i}\right)=p\left(N_{A}\right) / m$. The shape is then measured from the probability of the leaves $p\left(L_{j}\right)$, given a tree with $x$ leaves, by $-\sum_{j=0}^{x} p\left(L_{j}\right) \log _{2}\left(p\left(L_{j}\right)\right)$. Balance is measured as follows. Given all the possible values $V_{i}$ for $p\left(L_{j}\right)$, calculate $G\left(V_{i}\right)$ as $G\left(V_{i}\right)=n * V_{i}$ where $n$ is the number of times $V_{i}$ occurs in the set of all the leaves of the tree. The balance is then measured by sum $\sum_{j=0}^{x} G\left(V_{j}\right) \log _{2}\left(G\left(V_{j}\right)\right.$ for all the $x$ possible values for $p\left(L_{j}\right)$.

1 The system is a development of the one described in [1].

2 Notice that the meta-learner misclassifications might have different impact on the overall system performance.

${ }^{3}$ Notice that the meta-learning system itself assumes a simplicity bias. A meta-learner, as any learner, requires biases. 


\section{Experiments with pruning strategies}

\section{Learning builds daily accumulation, but the practice of pruning builds daily simplification.D'après Lao Tzu}

THE ENTRENCHER system can be applied to a simplicity bias pool to produce a learned bias classifier that can decide between different pruning options. Different pruning strategies have been proposed ${ }^{4}$. Quinlan's C4.5 system popularised error-based pruning. A major alternative to the error-based approach is the cost-complexity pruning approach that has been adopted by some decision tree learning systems including Quinlan's own ID3.

The cost-complexity pruning technique considered here, as Quinlan's errorbased strategy, admits of different levels of pruning according to the different values of the acceptable cost threshold. Pruned trees are generated by replacing subtrees by a leaf labeled by the majority class that covers all but exception $\left(E_{\text {majority }}\right)$ cases of the subtree coverage $\left(X_{\text {subtree }}\right)$, whenever the cost of the subtree is greater than the acceptable cost threshold. Let $D_{\text {subtree }}$ be the depth of a subtree, $D_{\text {tree }}$ the depth of the whole tree and $S_{\text {subtree }}$ the size in terms of the number of nodes of the subtree. Cost is then defined as Cost subtree $_{\text {stre }}=$ $\frac{\left(\left(D_{\text {tres }}-D_{\text {subtree }}+1\right)^{2}\right) * S_{\text {subtree }}}{E_{\text {majorty }} / X_{\text {subtree }}}$. Pruning proceeds from the leaves to the root of the tree.

The experiments reported in this paper involve learning tasks from an artificial domain consisting of MoNK-like problems [3]. Given the original 6 attributes of the MONK problems, there are $2^{4} 32$ possible classifications. For the current experiments, 100 classifications were randomly chosen and 10 problems, composed by training and a test sets of 50 instances, were constructed for each classification. The meta-learning system was trained on an increasing number of problems and then tested on different test sets of problems randomly drawn from the 1000 existing problems. The performances were compared to the best possible pruning option available for each problem and with the fixed different pruning options. In the graphs that report the experiments, the $X$-axis represents the training set sizes and the $Y$-axis the accuracy in the test sets of 50 problems. Each point represents the average of 10 runs under a fixed training set size.

\subsection{Learning when to prune}

It is clear that there are no grounds for believing that the simplest course of events will really happen. Ludwig Wittgenstein

For each pruning strategy considered, a first question to ask is when pruning will enhance performance. In the first experiment, the meta-learning system is applied to decide between pruning with confidence level of $25 \%$, C4.5's default value, and leaving the original C4.5 tree unpruned. In another experiment, the appropriateness of cost-complexity pruning has been considered. Here the metalearner decides between pruning with a fixed acceptable cost threshold of 150

${ }^{4}$ Refer to [5] for overview and an comparison. 
and leaving the original tree unpruned. Fig. 1 shows the result. The two bottom lines represent pruning and no pruning. In the case of cost-complexity, unpruned trees have the worse performance all along whereas in the error based pruning case, the bottomost line is the performance of pruned trees. In both cases the top line is the best pruning option for each problem and the second line approaching it is the performance of THE ENTRENCHER.
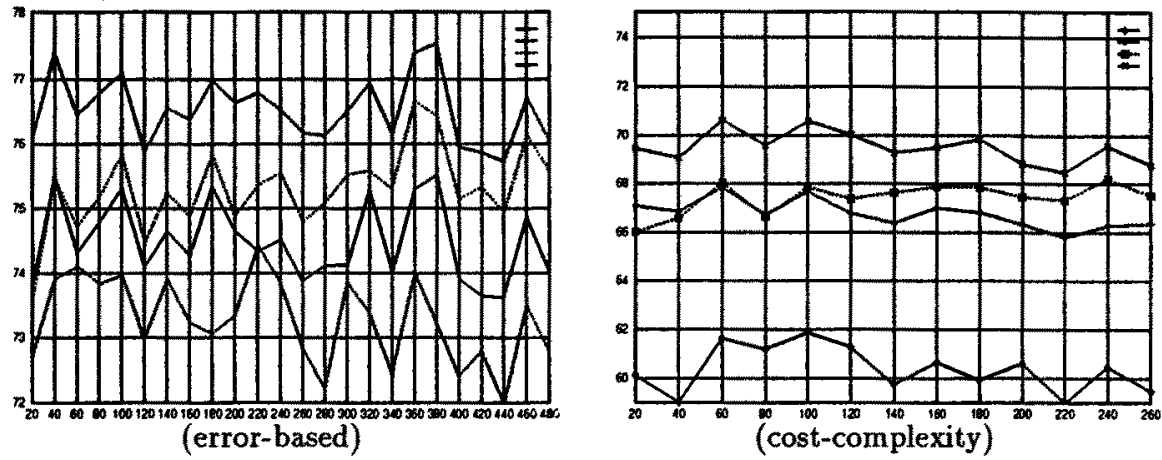

Fig. 1. Learning when to prune

\subsection{Learning how much to prune}

...as simple as possible but not simpler. Albert Einstein

Another question is how much simplicity is good enough. Fig. 2 plots the performance of the meta-learning system when selecting between different confidence levels for error-based pruning $(0,25,50,75)$. Fig. 3 plots the curves for selecting the amount of cost-complexity pruning that the 50 test problems require by considering different acceptable cost thresholds $(50,150,250)$.

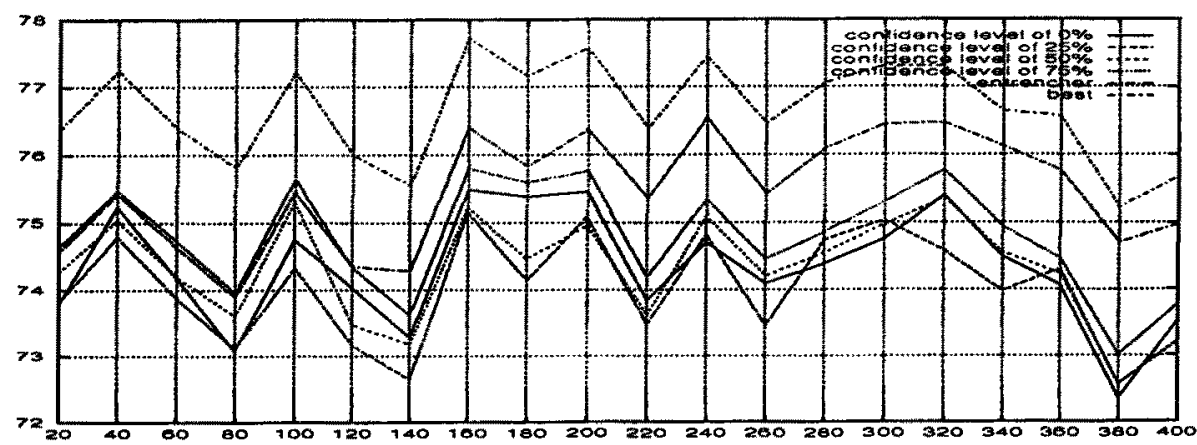

Fig. 2. Learning how much error-based pruning is required 


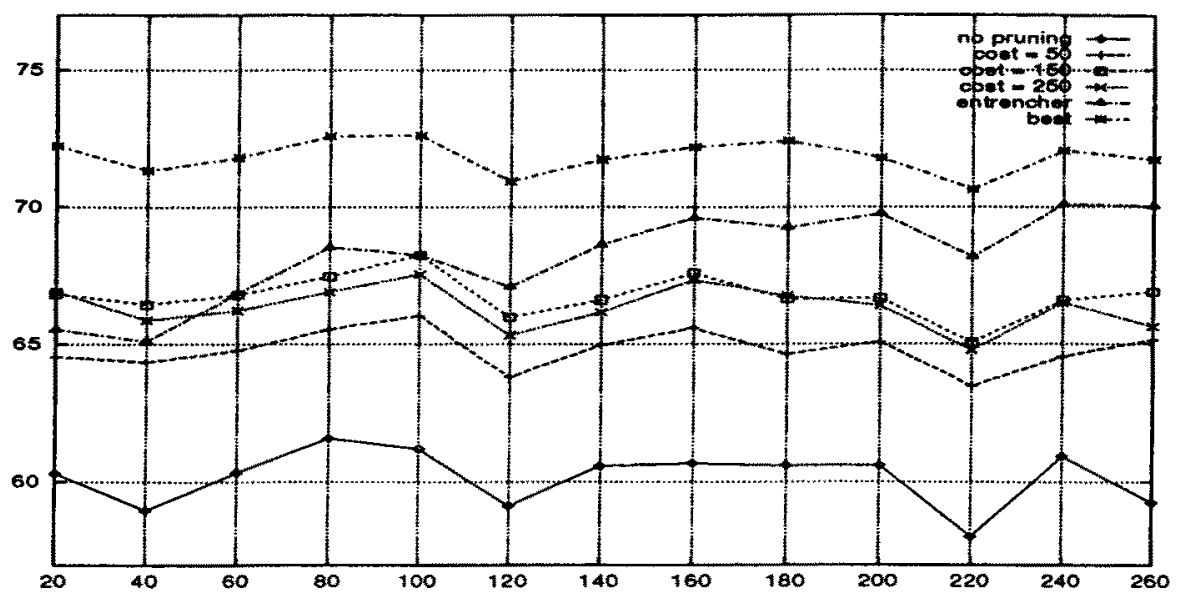

Fig. 3. Learning how much cost-complexity pruning is required

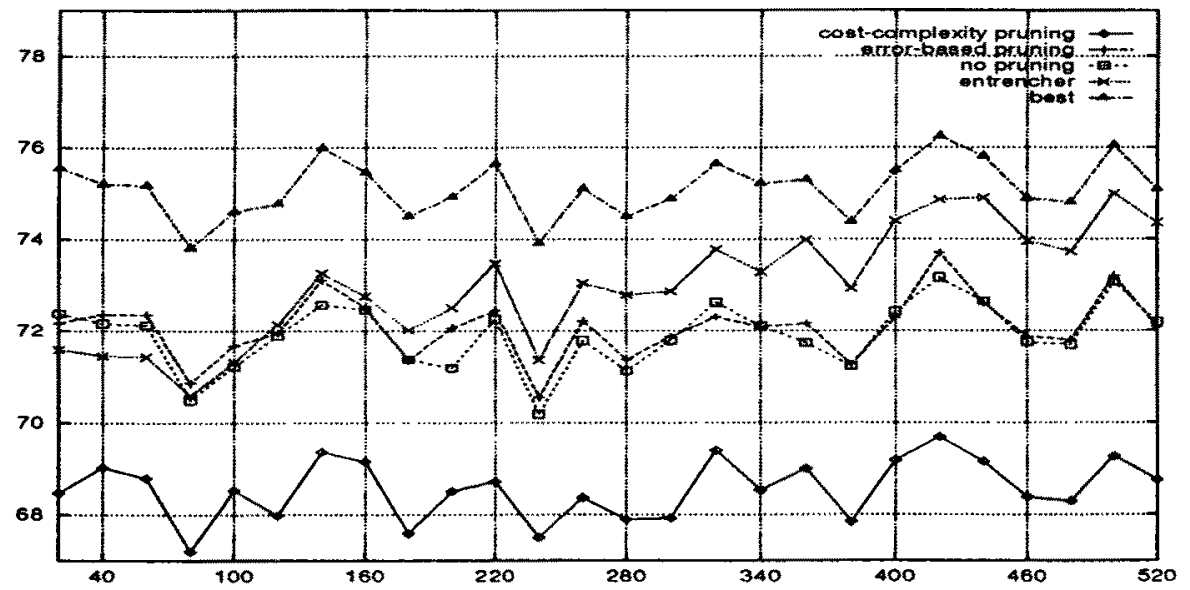

Fig. 4. Learning how to prune

\subsection{Learning how to prune}

One thing at least is certain [...], that the simple injunction "Simplify" is inadequate, if only because it is ambiguous. Mario Bunge

A third question concerns simplicity is related to the choice between different pruning strategies. An informed choice between C4.5 trees with no pruning, cost-complexity pruning with acceptable cost threshold of 150 and error-based pruning with confidence level of $25 \%$ can be provided by the use of THE ENTRENCHER, as Fig. 4 reports. 


\section{Conclusion}

Since simplicity is not a universal bias, the question is: How do we find out when, how much and how to apply it? The experiments in this paper show that THE ENTRENCHER allows for a successful (inductively) informed choice between different learning options as the system performance curves tend towards the best curve as training set size increases. It is therefore possible to learn about the appropriateness of different simplicity biases. Whitehead once said seek simplicity and distrust it. We should understand that since God doesn't always shave with an Occam razor and Occam razors don't come with user's manuals, we are left to grope with nothing but induction to aid us.

\section{References}

1. H. Bensusan and P. Williams. Learning to learn boolean tasks by decision tree descriptors. In M. V. Someren and G. Widmer, editors, Poster Papers - 9th European Conference on Machine Learning, pages 1-11. 1997.

2. P. Brazdil, J. Gama, and B. Henery. Characterizing the applicability of classification algorithms using Meta-Level Learning. In Francesco Bergadano and Luc de Raedt, editors, Proceedings of the European Conference on Machine Learning, volume 784 of $L N A I$, pages 83-102, Berlin, April 1994. Springer.

3. S. Thrun et al. The monk's problems. Technical Report CMU-CS-91-197, School of Computer Science, Carnegie-Mellon University., Pittsburgh, PA - USA, 1991.

4. D. Gamberger and N. Lavrac. Conditions for Occam's Razor applicability and noise elimination. In Marteen van Someren and Gerhard Widmer, editors, Proceedings of the 9th European Conference on Machine Learning, pages 108-123. Springer, 1997.

5. J. Mingers. An empirical comparison of pruning methods for decision tree induction. Machine Learning, 4:227-243, 1989.

6. P. Murphy and M. Pazzani. Exploring the decision forest: An emprical imvestigation of occam's razor in decision tree induction. Journal of Artificial Intelligence Research, 1:257-275, 1994.

7. J. R. Quinlan. C4.5: Programs for Machine Learning. Morgan Kaufmann, San Mateo, CA, USA, 1993.

8. G. Webb. Further experimental evidence against the utility of occam's razor. Journal of Artificial Intelligence Research, 4:397-417, 1996. 